Aus dem Balneologisch-hygienischen Institute in Marienbad. (Vorstand: Dr. Zörkendörfer.)

\section{Ueber Sulfatausscheidung beim Gebrauche alkalisch-salinischer Quellen.}

\section{Von Dr. Max Porges.}

Die Untersuchungen, über deren Resultat ich berichten möchte. stellen das Anfangsglied einer Versuchsreihe dar. welche dahin gehen soll, die Pharmakodynamik der Heilquellen unter Bedingungen zu studieren, wie sie ihrer zumeist auf Empirie beruhenden Anwendung an Ort und Stelle entsprechen. Die Schaffung eines balneologischen Instituts durch die Stadt Marienbad hat uns diese Möglichkeit eröffnet.

So soll hier vorerst über die Resorptions- und Ausscheidungsverhältnisse der Sulfate, wie sie in den Marienbader alkalisch-salinischen Heilquellen enthalten sind, berichtet werden.

In der Literatur findet sich über dieses Kápitel hauptsächlich die Arbeit von Wagner (1) und Buchheim (2) und eine Dissertation von Sick (3).

Auch Seegen (4) weist in einer kurzen Bemerkung auf die Sulfatausscheidung bei dem Gebrauche von Karlsbader Mühlbrunnen hin, doch steht das Resultat dieses Versuches mit demjenigen der übrigen Autoren in solchem Widerspruche, daß es wohl noch einer Nachprüfung bedürfte.

Wagner und Buchheim kommen auf Grund ihrer Versuche zu dem Schlusse: „Die abfïhrende Wirkung der Sulfate sei so zu denken, daß diese im Darmkanal wegen ihres geringen Diffusionsvermögens nur langsam resorbiert werden. daß sie daher mit ziemlich vielem Wasser in den unteren Teil des Darmkanals, welcher gewöhnlich einen konsistenteren Inhalt hat. gelangen und so aus diesem schnell entleert werden. Die beschleunigte peristaltische Bewegung ist rielleicht nur die Folge der Anwesenheit einer größeren Menge von fremdartigen Stoffen im unteren Teile des Darms. sodaß wir also nicht genötigt wären. eine eigentümliche Einwirkung jener Stoffe auf die Darmnerven anzunehmen."

Aus den neueren Arbeiten von Heidenhain, Hamburger. Hoeber und anderen (5) ist nichts über die Ausscheidungsverhältnisse der Schwefelsäure im Harn zu entnehmen. Diese Autoren haben, ebenso wie ältere Forscher, die Frage der Resorption dadurch zu lösen gesucht, daß sie die im Darme zurückbleibende Flüssigkeit in Untersuchung zogen.

In meinen Versuchen bin ich folgendermaßen vorgegangen:

Es wurde die gesamte Schwefelsäure des Harnes und der Faeces als Anhydrid während einer Vorperiode bestimmt. sodann w wärend der Versuchsperiode eine bestimmte Menge Marienbader Kreuzbrunnen gegeben, dessen Gesamtschwefelsäure in gleicher Weise bestimmt wurde, und dann eine kurze Nachperiode bis zum Absinken der Sulfate auf die Norm angeschlossen.

In allen Fällen handelte es sich um gesunde Individuen ohne Stoffwechselanomalie mit intakter Darmfunktion. Die Kost war in den ersten beiden Fällen eine gemischte, im Falle 3 eine vorwiegende Kohlehydraternährung. im Falle 4 eine vorwiegende Eiweißdiät. Dieser letzte Fall befand sich in Stickstoffgleichgewicht.

Die mit der Nahrung eingeführte Schwefelsäure zu bestimmen, erschien vollkommen überflüssig, da die Schwefelsäure des Harns normalerweise mindestens zum Teile nicht aus eingeführten Sulfaten, sondern aus anderen Schwefelverbindungen stammt. Außerdem wurde die normale Schwefelsäureausscheidung unserer Versuchspersonen in einer Forperiode, deren einzelne Tagesmengen gut übereinstimmende Werte ergaben, bestimmt und der Durchschnittswert, der sich erheblich von den Schwefelsäuremengen der Versuchsperiode unterschied, der Berechnung zugrunde gelegt.

Der Versuchsverlauf ergibt sich aus folgenden Tabellen, in welchen die Schwefelsäuremengen als $\mathrm{SO}_{3}$ berechnet angegeben sind.

\begin{tabular}{|c|c|c|c|c|c|c|c|c|c|c|}
\hline & Periode & $\stackrel{\infty}{m}$ & 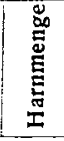 & 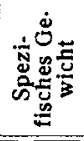 & 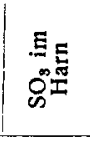 & 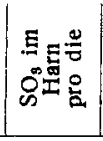 & 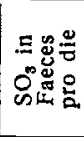 & 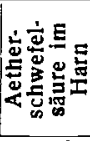 & 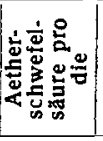 & 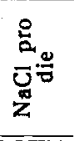 \\
\hline & Vorperiode & \begin{tabular}{|l}
1 \\
2 \\
3 \\
4 \\
5 \\
6 \\
\end{tabular} & $\begin{array}{r}740 \\
1600 \\
1100 \\
400 \\
1560 \\
1000 \\
\end{array}$ & $\begin{array}{l}1.030 \\
1.013 \\
1.023 \\
1.030 \\
1.015 \\
1.020 \\
\end{array}$ & $\begin{array}{l}2.1544 \\
1.6640 \\
2.5154 \\
1.4302 \\
2.1224 \\
1.9891 \\
\end{array}$ & 1.9794 & 0.018 & & \begin{tabular}{|l|l|} 
& 0.1467 \\
\end{tabular} & 9.09 \\
\hline & 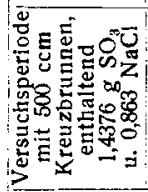 & $\begin{array}{l}1 \\
2 \\
3 \\
4 \\
5 \\
6 \\
7 \\
8 \\
\end{array}$ & $\begin{array}{l}1980 \\
1140 \\
990 \\
1060 \\
1610 \\
1260 \\
1875 \\
1620\end{array}$ & $\begin{array}{l}1.014 \\
1.024 \\
1.028 \\
1.027 \\
1.016 \\
1.026 \\
1.015 \\
1.018\end{array}$ & $\begin{array}{l}2.6635 \\
3.4152 \\
3.2781 \\
3.1260 \\
3.0629 \\
3.5992 \\
3.6285 \\
3.7310\end{array}$ & $\begin{array}{c}3.3123 \\
=66 \%\end{array}$ & 0.060 & & 0.1533 & 15.3 \\
\hline & $\begin{array}{c}\text { Nach- } \\
\text { periode }\end{array}$ & $\begin{array}{l}1 \\
2 \\
3 \\
\end{array}$ & $\begin{array}{l}1450 \\
1345 \\
1300 \\
\end{array}$ & $\begin{array}{l}1.020 \\
1.019 \\
1.021 \\
\end{array}$ & $\begin{array}{l}3.1671 \\
2.1059 \\
2.1131 \\
\end{array}$ & 2.1085 & 0.0013 & & 0.1545 & 12. \\
\hline & Vorperiode & \begin{tabular}{|l}
1 \\
2 \\
3 \\
4 \\
5
\end{tabular} & $\begin{array}{l}1170 \\
2640 \\
2940\end{array}$ & $\begin{array}{l}1.020 \\
1.021 \\
1.017\end{array}$ & $\begin{array}{l}.4654 \\
4.6522 \\
5.0203\end{array}$ & 2.4726 & 0.0078 & $\begin{array}{l}0.1249 \\
0.2679 \\
0.2261\end{array}$ & 0.1238 & \\
\hline & $\begin{array}{c}\text { Versuchs } \\
\text { periode } \\
600 \mathrm{~g} \text { Kreuz- } \\
\text { brunn. enth } \\
1.7893 \mathrm{~g} \mathrm{SO}_{3} \\
1.05 \mathrm{~g} \mathrm{NaCl}\end{array}$ & $\left.\begin{array}{l}1 \\
2 \\
3 \\
4 \\
5\end{array}\right\}$ & $\begin{array}{l}3000 \\
4420\end{array}$ & $\begin{array}{l}1.016 \\
1.020\end{array}$ & $\begin{array}{l}5.7541 \\
11.8735\end{array}$ & $\begin{array}{l}3.5255 \\
=45 \%\end{array}$ & 0.1222 & $\begin{array}{l}0.2781 \\
0.3520\end{array}$ & 0.1260 & 9.3 \\
\hline & $\begin{array}{c}\text { Nach- } \\
\text { periode }\end{array}$ & $\begin{array}{l}1 \\
2 \\
3\end{array}$ & $\begin{array}{l}1000 \\
3200\end{array}$ & $\begin{array}{l}1.026 \\
1.015 \\
\end{array}$ & $\begin{array}{l}9.9963 \\
3.6078 \\
\end{array}$ & 1.8039 & & 0.2571 & $\begin{array}{l}0.1285 \\
\end{array}$ & \\
\hline \multicolumn{2}{|r|}{$\mathrm{v}$} & 0 & $\begin{array}{l}1780 \\
2740 \\
3075\end{array}$ & $\begin{array}{l}1.021 \\
1.020 \\
1.021\end{array}$ & $\begin{array}{l}2.2004 \\
\mathbf{4}, 8970 \\
5.0461\end{array}$ & 2.4287 & 0.0011 & $\begin{array}{l}0.1552 \\
0.2753 \\
0.2702\end{array}$ & 0.1401 & 17.70 \\
\hline \multirow{2}{*}{\multicolumn{2}{|c|}{ 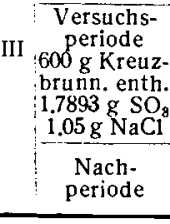 }} & $\begin{array}{l}1 \\
2 \\
3 \\
4 \\
5\end{array}$ & $\begin{array}{r}3640 \\
7180\end{array}$ & $\begin{array}{l}1.020 \\
1.017\end{array}$ & $\begin{array}{r}7.9234 \\
12.4431\end{array}$ & $\begin{array}{l}4.0753 \\
=67 \%\end{array}$ & 0.008 & $\begin{array}{l}0.2998 \\
0.4437\end{array}$ & 0.1447 & 16.9 \\
\hline & & $\left.\begin{array}{l}1 \\
2 \\
3\end{array}\right\}$ & $\begin{array}{l}2020 \\
4045 \\
\end{array}$ & $\begin{array}{l}1.020 \\
1.017 \\
\end{array}$ & $\begin{array}{l}2.6530 \\
5.4389 \\
\end{array}$ & 2.7194 & & $\begin{array}{l}0.1787 \\
0.2635 \\
\end{array}$ & 0.1317 & \\
\hline & Vorperiode & $5 \mathrm{~T}$. & 8500 & 1.018 & 13.3221 & 2.6664 & 0.002 & 0.5194 & 0.1039 & 13 \\
\hline & $\begin{array}{l}\text { Versuchs- } \\
\text { periode } \\
600 \mathrm{~g} \text { Kreuz- } \\
\text { brunn. enth. } \\
1.9136 \mathrm{~g} \mathrm{SO}_{3}\end{array}$ & $\stackrel{\sigma}{\sigma}_{0}^{\circ}$ & 13260 & 1.017 & 23.7623 & $\begin{array}{l}3.9604 \\
=48 \%\end{array}$ & 0.132 & 0.6537 & 0.1259 & 13 \\
\hline & $\begin{array}{l}\text { Nach } \\
\text { period }\end{array}$ & $2 \mathrm{~T}$. & & 1.016 & 6.9791 & 3.4895 & & 2456 & 0,1228 & 13.5 \\
\hline
\end{tabular}

Fall 1 zeigt in der Vorperiode eine durchschnittliche Sulfatausscheidung von $1.9794 \mathrm{~g}$ pro die im Harn und 0,018 in den Faeces während der Verabreichung von $500 \mathrm{~g}$ Kreuzbrunnen mit dem Sulfatgehalte 1,437 in der Versuchsperiode sehen wir ein Ansteigen der Sulfatausscheidung auf $3.3123 \mathrm{~g}$ pro die im Harn, was einer Steigerung von $66 \%$ entspricht. Hier ebenso wie in den übrigen Fällen sinkt die Menge der ausgeschiedenen Sulfate nicht sofort mit dem Sistieren der gesteigerten Zufuhr auf die Norm ab, sondern überdauert sie wie bei Buchheim und Wagner eine kurze Zeit.

Fall 2 zeigt ein wesentlich anderes Verhalten: Trotz erhöhter Sulfatzufuhr - $1.7898 \mathrm{~g}$ in $600 \mathrm{~g}$ Kreuzbrunnen gegenüber $1,4346 \mathrm{im}$ Falle 1 - beträgt hier die relative Steigerung nur $45 \%$, hingege weisen die Faeces eine erhebliche Zunahme an Sulfaten während der Versuchsperiode auf.

Ein gleiches Verhalten beobachten wir im Falle 3 und 4. Das. selbe ist sehr charakteristisch: Während nämlich im Falle 1 und 3 eine abführende Wirkung durch das eingeführte Mineralwasser nicht erzielt wurde, traten im Falle 2 und 4 die charakteristischsten nach Schwefelwasserstoff riechenden wässerigen Stühle auf.

Es spricht dies für die Richtigkeit der Buchheimschen Auffassung.

Im Falle 1 und 3 erscheinen die eingeführten Sulfate nahezu vollständig im Harn, sind also wohl bereits resorbiert, bevor sie im unteren Darm eine Wirkung auslösen können; im Falle 2 und 4 fehlt ein beträchtlicher Teil davon in dem Harn, erscheint jedoch in den Faeces, ist also der Resorption in den oberen Darmpartien entgangen und hat seine Wirkung am Ende des Darmrohres entfalten können. Dementsprechend weist Fall 3 eine Steigerung der Sulfatausscheidung im Harn um $67 \%$, Fall 4 nur um $48 \%$ auf. Die Ausscheidungsverhältnisse der Aetherschwefelsäure zeigen entgegen den Untersuchungen anderer Autoren keine nennenswerten Veränderungen. Allerdings hielten sich die Werte derselben in meinen Fällen überhaupt in physiologischen Grenzen.

Es wäre immerhin möglich, daß dort, wo ursprünglich eine Vermehrung der Aetherschwefelsäure stattfindet, die Zufuhr von Sulfaten ein Absinken zur Norm herbeiführen könnte.

Ueber die Resultate einer dahingehenden Versuchsreihe werde ich später berichten. 
Das Verhalten der Chloride zeigt nichts Charakteristisches.

Ich komme also auf Grund meiner Versuche zu folgendem Schlusse:

1. Die mit den alkalisch-salinischen Wässern eingeführten Sulfate werden zum Teil resorbiert und erscheinen im Harn; 2. Resorption und abführende Wirkung stehen in einem antagonistischen Verhältnisse; 3 . die Aetherschwefelsäure wird bei an und für sich normaler Ausscheidung nicht beeinflußt.

Die Tatsache dieses Antagonismus zwischen Resorption und eccoprotischer Wirkung der Sulfate gibt uns einen Fingerzeig für die therapeutische Anwendungsweise der alkalischsalinischen Wässer, indem es unser Bestreben sein muß, dort, wo wir eine abführende Wirkung erzielen wollen, die Resorption tunlichst zu verzögern.

Ich möchte nicht verfehlen, zum Schlusse dem Herrn Kollegen Dr. Zörkendörfer für seine freundliche Unterstützung meinen besten Dank auszusprechen.

Literatur: I. Wagner, De effectu natri sulfurici. Inauguraldissertation. Dorpat 1853. - Buch heim, Ueber die Wirkung des Glaubersalzes. Archiv für physiologische Heilkunde Bd. 13, 1854. - 3. Sick, Versuche über die Abhängigkeit des Schwefelsäuregebaltes des Urins von der Schwefelsäurezufuhr. Inauguraldissertation. Tübingen 1859. - 3. Se egen, Physiologisch-chemische Untersuchungen über den EinfluB des Karlsbader Mineralwassers auf einige Faktoren des Stoffwechsels. Wiener medizinische Wochenschrift 1860. - 5. S e egen, Physiologische Untersuchungen über den Einfius des Glaubersalzes auf einige Faktoren des Stoffwechsels. Sitzungsberichte der Kaiserlichen Akademie der Wissenschaften. Wien, Bd. 49, Abt. 2. - Hamburger, Osmotischer Druck und Ionenlehre Bd. 2. Bergmann, Wiesbaden 1904. 